PROCEEDINGS OF THE

AMERICAN MATHEMATICAL SOCIETY

Volume 131, Number 7, Pages 1977-1980

S 0002-9939(03)06975-2

Article electronically published on February 11, 2003

\title{
FLATNESS AND THE ORE CONDITION FOR RINGS
}

\author{
PETER TEICHNER
}

(Communicated by Lance W. Small)

\begin{abstract}
We prove the following result on the universal localization of a ring $R$ at an ideal $I$ : If the universal localization is flat as an $R$-module, then $R$ satisfies the Ore condition with respect to the multiplicative set of elements that become invertible modulo $I$. It is well known that for domains the converse of this result holds, and hence we have found in this case a new characterization of the Ore condition.
\end{abstract}

\section{INTRODUCTION}

Why would a low-dimensional topologist be interested in localizations of noncommutative rings? First of all, unlike in higher dimensions, it is impossible to assume anything too drastic about the fundamental group $\pi_{1} M$ of, say, a 3dimensional manifold $M^{3}$, since this would actually answer most of the questions right away (modulo things like the Poincaré conjecture). Thus one is stuck with the usually noncommutative group ring $\mathbb{Z}\left[\pi_{1} M\right]$. The homotopy and homology groups of $M$ become modules over this ring, hence K-theory enters the picture. As for L-theory, there are linking forms defined on the first homology of covers of $M^{3}$; see e.g. 3. If $\pi$ is the deck transformation group of the cover, then these linking forms take values in the quotient $S^{-1} \cdot \mathbb{Z}[\pi] / \mathbb{Z}[\pi]$ which in the easiest case $\pi=1$ could be the usual coefficient group for linking forms $\mathbb{Q} / \mathbb{Z}$. The multiplicative set $S$ which defines the above localization is quite flexible and is usually chosen according to desired applications. For example, the complement of a knot $K$ in 3-space has a canonical infinite cyclic cover $M(K)$. Then the Alexander polynomial $A_{K}(t)$ of the knot is nothing else than the characteristic polynomial of $H_{1}(M(K))$ as a $\mathbb{Z}[\pi]$-module, with $\pi$ infinitely cyclic, generated by $t$. Moreover, the resulting linking form, the "Blanchfield pairing", takes values in $\mathbb{Q}(t) / \mathbb{Z}\left[t, t^{-1}\right]$ where $\mathbb{Q}(t)$ is the quotient field of the group ring. It gives additional information about the knot $K$ known as "S-equivalence", like the twisted signatures or twisted Arf invariants of the knot.

In [1] we generalize this example from $\mathbb{Z}$ to other torsionfree solvable groups $\pi$. It is interesting to observe that unless the Alexander polynomial is trivial, i.e. $\pi_{1} M(K)$ is perfect, the commutator series of the fundamental group of the knot complement never stabilizes, providing a huge number of geometrically interesting torsionfree solvable groups. For such a group, the nonzero elements of $\mathbb{Z}[\pi]$ satisfy

Received by the editors July 5, 2001.

2000 Mathematics Subject Classification. Primary 16S10.

This research was supported by the NSF, grant DMS0072775.

(C)2003 American Mathematical Society 
the Ore condition (see below) which implies that there is a skew-field of classical quotients for our (regular) group ring. It is extremely important in this particular application that this Ore localization is flat as a module over $\mathbb{Z}[\pi]$, otherwise many of the homological calculations go awry.

It was this desire for flatness that tempted the author to study which other localizations could have this important property. It turns out that for the most interesting multiplicative sets, flatness actually implies the Ore condition!

Main Theorem. If the universal localization of a ring $R$ at a two-sided ideal $I$ is flat as a left $R$-module, then $R$ satisfies the right Ore condition with respect to the multiplicative set of elements that become invertible modulo $I$.

Many thanks to Andrew Ranicki and Lance Small for discussions on the topic. In particular, Lance pointed out that the above theorem does not hold for more general multiplicative sets, justifying our special assumption (which was originally motivated from the topological applications). In the example one takes a field $\mathbb{K}$ and the ring

$$
R:=\left(\begin{array}{cc}
\mathbb{K} & 0 \\
\mathbb{K}(x) & \mathbb{K}[x]
\end{array}\right), \quad S^{-1} R=\left(\begin{array}{cc}
\mathbb{K} & 0 \\
\mathbb{K}(x) & \mathbb{K}(x)
\end{array}\right)
$$

with multiplicative set $S$ equal to all nontrivial elements in $R$. Then $S$ satisfies the left Ore-condition, thus the above quotient field exists (and is isomorphic to the universal localization). However, $S$ does NOT satisfy the right Ore-condition but $S^{-1} R$ is still flat as a left $R$-module.

\section{ORE'S CONDITION}

Let $R$ be a ring with unit. For any subset $S$ of $R$ one has the universal $S$-inverting localization $R \rightarrow R_{S}$, i.e. the elements of $S$ become invertible in $R_{S}$ and if that is also the case for another ring-homomorphism $R \rightarrow A$, then there is a commutative diagram

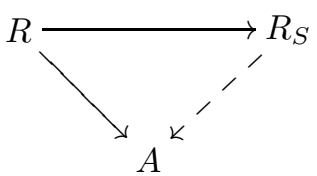

with a unique map $R_{S} \rightarrow A$. Then $R_{S}$ is well defined up to canonical isomorphism, and one gets the same result if one closes $S$ multiplicatively; see e.g. 22. The ring theoretic properties of $R_{S}$ are far from being understood. In general, one does not even have a good criterion for garanteeing the nontriviality of $R_{S}$.

A particularly good case is when $R$ is commutative and $S$ contains no zerodivisors. Then the ring $R_{S}$ consists of fractions with denominators from $S$ and many properties of $R$ carry over to $R_{S}$. Moreover, $R_{S}$ is flat as an $R$-module which is a key property in many homological calculations.

In the noncommutative context one needs two conditions for an analogous ring of fractions to exist. One condition has to do with zero-divisors in $S$ and the other is as follows.

Ore's condition. For every $r \in R$ and $s \in S$ the intersection $s R \cap r S$ is nonempty.

The explanation for this condition is simple: If every element is supposed to be of the form $r \cdot s^{-1}$, then in particular one has to find $r_{0}, s_{0}$ such that $s^{-1} \cdot r=r_{0} \cdot s_{0}^{-1}$ for every given $r \in R$ and $s \in S$. This is obviously equivalent to the above condition 
(which has the crucial advantage of being formulated inside $R$ ). Note that one can interchange the order of ring elements, so that the above is really the right Ore condition. We will suppress this distinction except for the precise statement of our Main Theorem below.

Theorem (Ore [4). If $S$ contains no zero-divisors and satisfies the Ore condition, then $R_{S}$ is isomorphic to the Ore localization $R \cdot S^{-1}$ which by definition consists of pairs $(r, s) \in R \times S$ modulo a concrete equivalence relation which reflects the fact that $(r, s)$ really denotes $r \cdot s^{-1}$.

The Ore localization has many very good ring theoretic properties; see [5] or [2]. In particular, as in the commutative case, $R \cdot S^{-1}$ is flat over $R$. This fact is well known if $S$ contains no zero-divisors; see e.g. [5, Prop. 3.5]. However, it seems worth pointing out that flatness really has nothing to do with zero-divisors. Since we have not found this simple fact in the literature, we give a short proof.

Proposition 1. If $R$ satisfies the Ore condition with respect to $S$, then the universal localization $R_{S}$ is flat as a left $R$-module.

Proof. The important observation is that the Ore condition implies that every element of $R_{S}$ can be written in the form $r \cdot s^{-1}$ with $r \in R$ and $s \in S$ (even though the relations on these fractions can be complicated in the presence of zerodivisors). Moreover, one has "common multiples", i.e. given finitely many $s_{i} \in S$, there is an $s \in S$ such that $s_{i}^{-1} \cdot s \in R$. More precisely, this means that there are $r_{i} \in R$ such that $s:=s_{i} \cdot r_{i} \in S$.

Now let $i: M \rightarrow N$ be a monomorphism of right $R$-modules. We want to show that

$$
i \otimes \mathrm{id}: M \otimes_{R} R_{S} \longrightarrow N \otimes_{R} R_{S}
$$

is still a monomorphism. By the above observations, an arbitrary element $x \in M \otimes$ $R_{S}$ can be written as $x=m \otimes s^{-1}$ with $m \in M$ and $s \in S$ : Clearly, $x=\sum_{i} m_{i} \otimes s_{i}^{-1}$ but by using a common multiple $s:=s_{i} \cdot r_{i} \in S$ we get

$$
x=\sum_{i} m_{i} \otimes s_{i}^{-1}=\sum_{i} m_{i} \otimes r_{i} \cdot s^{-1}=\sum_{i} m_{i} \cdot r_{i} \otimes s^{-1}=: m \otimes s^{-1} .
$$

If $x \in \operatorname{Ker}(i \otimes \mathrm{id})$, then

$$
0=(i \otimes \mathrm{id})(x) \cdot s=\left(i(m) \otimes s^{-1}\right) \cdot s=i(m) \otimes 1 .
$$

\section{Flatness}

We now restrict our attention to a special class of multiplicative subsets. Namely, let $I$ be a two-sided ideal in $R$ and let

$$
S_{I}:=\{r \in R \mid r \text { becomes invertible } \bmod I\} .
$$

In this case we let $R_{(I)}:=R_{S_{I}}$, the universal localization at $I$. Then the main result stated in the Introduction is the following characterization of the Ore condition.

Main Theorem. Let $R$ and $I$ be as above. Then $R$ satisfies the right Ore condition with respect to $S_{I}$ if and only $R_{(I)}$ is flat as a left $R$-module. 
Proof. We first need a slight reformulation of the Ore condition for general $S$. For $r_{i} \in R$ define the right $R$-ideal

$$
\left(r_{1}: r_{2}\right):=\left\{a \in R \mid r_{2} \cdot a \in r_{1} R\right\} .
$$

Lemma 1. The Ore condition is equivalent to $(s: r) \cap S \neq \emptyset$ for every $r \in R$ and $s \in S$.

The following lemma will be applied in particular for the right ideals $(s: r)$ above.

Lemma 2. For any right ideal $J$ in $R$ the two conditions $J \cap S_{I} \neq \emptyset$ and $R / J \otimes_{R}$ $R / I=0$ are equivalent. (Here $R / J$ and $R / I$ are viewed as right respectively left $R$-modules in the natural way.)

Proof. Since the tensor product functor $\otimes_{R} R / I$ is right exact, we have an exact sequence

$$
J \otimes_{R} R / I \stackrel{f}{\longrightarrow} R / I \longrightarrow R / J \otimes_{R} R / I \longrightarrow 0
$$

where $f(j \otimes r) \equiv j \cdot r \bmod I$. This implies that $R / J \otimes_{R} R / I=0$ if and only if there are $j_{k} \in J$ and $r_{k} \in R$ such that $\sum_{k} j_{k} \cdot r_{k} \equiv 1 \bmod I$. Since $J$ is a right ideal this condition is equivalent to the existence of a $j \in J$ such that $j \equiv 1 \bmod I$. But this is clearly equivalent to $J \cap S_{I}$ being nonempty.

To finish the proof of our theorem, we need to show that for $r \in R$ and $s \in S$ we have $R /(s: r) \otimes_{R} R / I=0$. By the universal property, we have a surjection $R_{(I)} \rightarrow R / I$ of rings and thus it suffices to show that

$$
R /(s: r) \otimes_{R} R_{(I)}=0 .
$$

Note that left multiplication with $r$ gives an exact sequence of right $R$-modules

$$
0 \longrightarrow(s: r) \longrightarrow R \stackrel{r .}{\longrightarrow} R / s R
$$

which implies that $R /(s: r)$ is isomorphic to a right $R$-submodule of $R / s R$. Since $R / s R \otimes_{R} R_{(I)}$ is generated by elements of the form $1 \otimes x$ with $x \in R_{(I)}$ (by the right exactness of the tensor product) it follows from the fact that $s \in S$ is invertible in $R_{(I)}$ that

$$
1 \otimes x=1 \otimes s \cdot s^{-1} \cdot x=s \otimes s^{-1} \cdot x=0
$$

and hence $R / s R \otimes_{R} R_{(I)}=0$. However, if $R_{(I)}$ is flat as a left $R$-module, then our submodule $R /(s: r)$ inherits this property which we needed to show.

\section{REFERENCES}

[1] T. Cochran, K. Orr and P. Teichner, Knot concordance, Whitney Towers, and $L^{2}$-signatures, Preprint 1999, math.GT/9908117; to appear in Annals of Math.

[2] P. Cohn, Skew Fields, Encyclopedia of Math., Vol. 57, Cambridge Univ. Press 1995. MR 97d:12003

[3] J. Duval, Forme de Blanchfield et cobordisme d'entrelacs bords, Comm. Math. Helv. 61, 617635, 1986. MR 88a:57037

[4] O. Ore, Linear equations in non-commutative fields, Annals of Math. 34, 480-508, 1931.

[5] B. Stenström, Rings of Quotients, Grundlehren der math. Wissenschaften, Vol. 217, SpringerVerlag 1975. MR 52:10782

Department of Mathematics, University of California at San Diego, La Jolla, CalIFORNIA 92093-0112

E-mail address: teichner@math.ucsd.edu 